\title{
Iontophoretic delivery of transdermal patches containing ropivacaine: sustaining the anesthetic effect in children
}

\author{
Tao Yu', Shengjie Zhang ${ }^{1}, \mathrm{Xu} \mathrm{Cao}^{2}$ and Chengyao Liu ${ }^{2}$ \\ 1Department of Operating Room Anesthesiology, Peking Union Medical College Hospital, Beijing Municipality, 100730, PR China; ${ }^{2}$ Department \\ of Otolaryngology-Head and Neck Surgery, Beijing TongRen Hospital, Capital Medical University, Beijing Municipality, 100730, PR China
}

\begin{abstract}
Clinical trial presented here investigates the sustaining anesthetic effect of ropivacaine when administered as an epidural injection followed by a transdermal patch with and without Reverse electrodialysis (RED) system in order to maintain anesthesia in children. It also focuses on the rate of release of the drug in vitro when administered with the RED system. The transdermal patch of ropivacaine was prepared using the evaporation technique enclosing $25 \mathrm{mg}$ of local anesthetic ropivacaine. Thus prepared patches were evaluated for physical parameters, such as folding endurance, tensile strength, in vitro drug release etc. The thicknesses of the patches were in the range of $0.02 \pm 0.006$ to $0.04 \pm 0.007 \mathrm{~mm}$, with $95.79 \pm 1.85$ to $97.45 \pm 0.07 \%$ drug content. The average weight and tensile strength were found to be $0.15 \pm 0.2$ to $0.17 \pm 0.18 \mathrm{gm}$, and $0.42 \pm 0.006$ to $0.58 \pm 0.002 \mathrm{~kg} /$ $\mathrm{cm}^{2}$. The folding endurance of the patches was in the range of $155.21 \pm 0.2$ to $167 \pm 0.29$ and the moisture content was $1.43 \pm 0.23 \%$ to $2.97 \pm 0.23 \%$. The formulation shows in vitro release in a phosphate buffer $(\mathrm{pH} 7.4)$ as $55.56 \%$ to $89.23 \pm 0.24 \%$ in $24 \mathrm{~h}$ with reverse electrodialysis system attached, and $43.76 \%$ to $81.23 \pm 0.14 \%$ in $24 \mathrm{~h}$ without reverse electrodialysis system. The children of different age groups, different body weight with no premedication of anesthetic or analgesic were selected as subjects with written informed consent and divided into two groups depending upon the RED system to be attached or not. The patches with and without the RED system were attached to the forearm of subjects and the trial was run. The results obtained demonstrate when attached with the reverse electrodialysis system, the patch had shown a greater potential for thermal threshold, lowering the cool sensation and the depth of pain.
\end{abstract}

Key words: child anesthesia, iontophoresis, reverse electrodialysis, ropivacaine, transdermal patch

Received: 19 September, 2018; revised: 03 March, 2019; accepted: 11 March, 2019; available on-line: 15 April, 2019

凶e-mail: bjtryylcy@sina.com

Acknowledgements of Financial Support: This project was financially supported by the Union Hospital mid issue XHHLKY201511 Abbreviations: AEM, anion exchange membrane; CEM, cation exchange membrane; HPMC, hydroxy propyl methyl cellulose; IP, iontophoresis; RED, reverse electrodialysis; RPV, ropivacaine; PAMAM, poly amido amine; PVA, polyvinyl alcohol; TDDS, transdermal drug delivery system; UV, ultra voilet

\section{INTRODUCTION}

Delivery of a drug via the topical route has always imposed a challenge for researchers, and the complex structure of the skin is the prime reason for this problem. One of the layers of the skin, known as the stratum corneum, serves as the toughest barrier for most of the molecules (Tyle, 2004). Despite that fact, the transdermal drug delivery system (TDDS) is one of the favorite systems to use due to its several clinical features, such as avoiding first-pass metabolism, being pain-free and self-dosing, as well as maintaining a steady-state plasma concentration. It also overcomes drawbacks due to oral administration, such as the $\mathrm{pH}$ and hindrance due to the presence of food or enzymes; clearly, in the case of topical administration, the gastrointestinal transit time can be eliminated. Highly lipophilic drugs with low molecular weight are the most suitable candidates for TDDS. Many attempts have been done in order to overcome the resistance of skin barriers (Sachanand et al., 2013). Till date, the most commonly used systems for topical delivery were creams and ointments used in various diseases. To be precise, large numbers of topically applied drugs are included in the transdermal drug delivery system to deliver an active ingredient into systemic circulation. The system provides an advantage in the controlled and painless release of the drug. The transdermal patch is one of the media adhering to the skin and used to deliver drug through the skin (Jett, 2018).

Transdermal patch, also known as a skin patch, is a medicated device placed on the skin to deliver a specific amount of enclosed drug through the skin layers into the bloodstream. TDDS in form of patches helps to deliver a drug at acontrolled, as well as predetermined, rate (https://en.wikipedia.org/wiki/Transdermal_patch). The mechanism of drug delivery through such a patch seems very simple. The drug is enclosed as a drug reservoir inside a patch which is applied to the skin surface for a definite period of time. The drug reaches the bloodstream via a diffusion mechanism where it maintains a steady-state plasma concentration. The method leads to a reduced pharmaceutical dosage as shortened metabolic pathway, when compared to a drug administered via the gastrointestinal route. However, there are slight limitations, as failure to maintain high dose levels, itching and sensitization of skin can happen (Mazzenga et al., 1991). The patch can be either a porous membrane or of a reservoir type. These patches are now proving to be a good alternative to for the existing therapeutic systems (Rawat et al., 2008).Various methods have been developed to deliver drugs via the transdermal route. Among them, iontophoresis (IP) is one of the best methods which work by exerting their electrical effects on the skin, the stratum corneum and also below layers of the skin.

The transdermal drug delivery involves transfer of charged ions, using electrical current, as a medium to cross biological barriers and various tissues, and this method is called iontophoresis (IP). The externally applied electrical potential results in an increase in ionic 
movement across biological membranes (William et al., 1998). The applied current is usually of voltage 0.1-5 V and a constant current of $0.5 \mathrm{~mA} / \mathrm{cm}^{2}$ for a predetermined specific period of time which causes transfer of the drug molecule or its salt across the skin barriers. The total amount of drug dislodged or transported is mostly dependent upon the concentration of the drug, the ionic charge, the intensity of applied voltage, and the surface area of contact with the device (Reeves et al., 2010). When the current is applied through biological membranes, the system is known as transdermal iontophoresis.

Transdermal iontophoresis consists of two oppositely charged electrodes applied on the skin surface which drive the drug molecule carrying a similar charge as an active electrode by repulsion across the skin. The amount of the drug delivered across the skin is directly proportional to the applied electrical charge and duration of the application (William et al., 1998).

Ropivacaine (RPV) is an amide-type local anesthetic agent whose s-isomeric form is active and is reported to have a similar therapeutic, as well as chemical properties to those of Bupivacaine (Cederthoim, 1997). RPV produces lower toxicity to the cardiovascular and the central nervous system when compared to the bupivacaine after intravenous infusion. In healthy human volunteers, RPV showed much less central nervous system symptoms and was found to be at least $25 \%$ less toxic when compared to bupivacaine (Chen et al., 2015; Daniele et al., 2008; Veerman et al., 2009). RPV had shown a distinct separation of the motor and sensory blocks due to the slightly lower lipid solubility in clinical trials and also had allowed an earlier motor blockade recovery in relation to bupivacaine. Due to the aforementioned advantages, RPV is well suited for the post-operative analgesia (Veerman et al., 2009).

The study presented here attempts to evaluate the effect of ropivacaine as a local anesthetic in children, using a reverse electrodialysis technique with the iontophoretic patch. Reverse electrodialysis (RED) is a technique which produces energy from a salinity gradient by contacting waters with different salinity, such as the sea water or river water, via a controlled mixing of these two solutions through an ion exchange membrane (Pattle, 1954; Post et al., 2008; Amnuaikit et al., 2005; Kanabar et al., 2015; Sleigh et al., 2014). The assembly of a typical RED system consists of membranes of ions (both cation and anion) whose movement is directed by the salinity gradient. As the membrane is favorable for passage of specific ions, cations and anions are transported in the opposite directions which gives rise to the net potential difference. Electroneutrality can be maintained due to a redox reaction that takes place at the electrode (Green, 2000; http://www.redstack.nl/en/technology/reverseelectrodialysis-red). The flow of electrons from the an- ode to the cathode via external circuit generates an external energy consumer.

The simple principle used here can be stated as that an ionic compound, like salt, gets separated into ions of positive or negative charge when it dissolves into water. The RED system uses an ion-exchange membrane to separate the fresh and salt water and to control the mixing of both. When the salt water is allowed to pass through the membrane, the ions will passively diffuse from the salt water to the fresh water where membrane acts as a selective barrier for ions. Positively charged ions will diffuse through the cation exchange membrane (CEM) while negatively charged ions will diffuse from the anion exchange membrane (AEM) which causes bulk transport and this opposing transport of ions creates positive and negatively charged poles like a battery or more precisely like the electrochemical cell.

The study presented here deals with formulation of a transdermal patch applied using an electrical impulse via reverse electrodialysis system followed by epidural injection of ropivacaine in order to maintain the level of anesthesia in children for $24 \mathrm{~h}$.

\section{MATERIALS AND METHODS}

\section{Materials}

Ropivacaine was purchased from the Shouguang $\mathrm{Fu}-$ kang Pharmacy Factory (Shandong, China); Chitosan and hydroxypropyl methylcellulose were purchased from Sigma Aldrich, USA; and other chemicals (polyvinyl alcohols (PVA), dibutyl phthalate, acetic acid, methanol) were purchased from the Shanghai Chemical Co. (Shanghai, China). The reverse electrodialysis device was purchased from REDstack, Netherlands.

\section{Methods}

Formulation of sterile injection of ropivacaine (Carolina et al., 2007). The sterile formulation of ropivacaine hydrochloride was prepared (Table 1) under aseptic conditions in a sterile area. Ropivacaine hydrochloride was added to $0.9 \% \mathrm{w} / \mathrm{v}$ solution of sodium chloride made in sterile water for injection. Propylene glycol was added in various concentration ranges as $2.5 \%, 3 \%$, $3.5 \%$, and $4 \%$ in order to enhance the solubility characteristic of ropivacaine. The final $\mathrm{pH} /$ tonicity of the system was adjusted using sodium hydroxide and/or hydrochloric acid to maintain $\mathrm{pH}$ between 4.0 to 6.0. Thus prepared formulation was evaluated for $\mathrm{pH}$, clarity and assay.

Preparation of ropivacaine patches using a solvent casting evaporation technique. The transdermal ropivacaine patch for reverse electrodialysis was prepared

Table 1. Formula composition of Ropivacaine hydrochloride Injection batches

\begin{tabular}{|c|c|c|c|c|}
\hline Ingredient & $\mathrm{R} 1$ & $\mathrm{R} 2$ & R3 & R4 \\
\hline Ropivacaine hydrochloride & $0.2 \%$ & $0.2 \%$ & $0.2 \%$ & $0.2 \%$ \\
\hline Sodium chloride & $0.9 \%$ & $0.9 \%$ & $0.9 \%$ & $0.9 \%$ \\
\hline Sodium hydroxide & q.s. & q.s. & q.s. & q.s. \\
\hline Hydrochloric acid & q.s. & q.s. & q.s. & q.s. \\
\hline Propylene glycol & $2.5 \%$ & $3 \%$ & $3.5 \%$ & $4 \%$ \\
\hline Sterile water for injection & q.s.to $100 \%$ & q.s.to $100 \%$ & q.s.to $100 \%$ & q.s.to $100 \%$ \\
\hline
\end{tabular}


using a solvent casting evaporation technique (Table 1), with the use of a glass petri plate (Kanabar et al., 2015). PVA, 4\%, was made to dissolve in distilled water by heating at $40^{\circ} \mathrm{C}$. The membrane was cast by pouring PVA solution into a petri dish, followed by drying in a hot air oven at $55^{\circ} \mathrm{C}$ for 8 hours. To prepare the drug reservoir compartment, HPMC was dissolved in double distilled water. To this solution, dibutyl phthalate was added as plasticizer and PAMAM dendrimer. This is labeled as solution A. Ropivacaine, dissolved in methanol, was added dropwise to this solution A with constant but slow stirring on a magnetic stirrer. Thus prepared dispersion was set on the PVA backing membrane and dried at $50^{\circ} \mathrm{C}$ for $5 \mathrm{~h}$. The chitosan membrane, used here as the rate controlling membrane, was prepared by dissolving chitosan to make a $2 \%$ in acetic acid, and cast on the drug reservoir. The whole patch was then dried, cut into small pieces and desiccated for further analysis.

Evaluation of ropivacaine hydrochloride injection. The formulated injection was evaluated for various parameters, such as clarity, $\mathrm{pH}$ and assay. The $\mathrm{pH}$ was checked using a digital $\mathrm{pH}$ meter. The percentage drug content was measured and calculated by using UV spectrometry. The solution was poured into a transparent container and observed visually against the dark and white background.

++ : Clear

+++ : Clear and transparent

Evaluation of transdermal ropivacaine patch for reverse electrodialysis. Physical characteristics. Five random patches were selected for this test. The smoothness, colour, and clarity of a patch were observed visually for each patch. Five random locations were selected for each patch and thickness was measured using a Vernier caliper. The average thickness was calculated. 10 different patches were randomly selected from the same batch, weighed and the average weight was determined.

Folding endurance. This test is performed to determine the strength of the patch, as well as to check the efficiency of plasticizer. Here, repeated folding of the patch was done until it broke up. The number of times the patch can be folded at the same place without getting cracked or broken is the value of folding endurance for that patch (Kanabar et al., 2015; Sleigh et al., 2014).

Drug content uniformity in the patch. An accurately weighed portion of the film (about $100 \mathrm{mg}$ ) is dissolved in $50 \mathrm{ml}$ of methanolic solution in which the drug is soluble, and then the solution is shaken continuously for $24 \mathrm{~h}$ in a shaker incubator. Next, the whole solution is sonicated. After sonication and subsequent filtration, concentration of the drug in solution is estimated spectrophotometrically at $265 \mathrm{~nm}$ by appropriate dilutions (Sleigh et al., 2014).

Tensile strength. This test gives an idea about mechanical properties and strength of patches. Universal strength testing apparatus (Hounsfield, Slinfold, Horsham, U.K.) was used to measure the tensile strength of the patch (Kanabar et al., 2015; Sleigh et al., 2014).

Percentage of moisture absorption and percentage moisture loss. 10 randomly selected patches were selected, weighed (W1/initial weight) and dried in a desiccator. After 5 days, these patches were reweighed (W2/ final weight) and the difference between W1 and W2 was calculated, which is percentage moisture loss. To determine percentage moisture loss the same procedure is repeated except that the patches are kept in a desiccator with calcium chloride. The difference in initial and final weight is measured (Kanabar et al., 2015).

In vitro skin permeation study. In vitro skin permeation study was performed using excised pig skin and a modified glass cell to which the skin section was attached, such that the contacting surface area was 12.19 $\mathrm{cm}^{2}$ and the glass tube (as the donor compartment) was dipped into a glass beaker containing isotonic phosphate buffer ( $\mathrm{pH}$ 7.4) which acts as a receptor compartment. $1 \mathrm{~cm}^{2}$ patch was placed on the membrane. Constant temperature was maintained at $37 \pm 0.5^{\circ} \mathrm{C}$ with stirring $(600 \mathrm{rpm})$ on a magnetic stirrer. For reference, the setups were categorized into two groups: passive diffusion and RED. For the RED set up, an $\mathrm{Ag} / \mathrm{AgCl}$ wire (anode) was dipped in the receptor compartment, while a conductive carbon film (4 $\mathrm{cm}^{2}$, cathode) was applied to the skin membrane. Both electrodes were connected to the RED cells, which were activated by adding $2 \mathrm{ml}$ of $11 \mathrm{mM}$ sodium chloride electrolyte solution. The donor compartment for each set was covered with parafilm to avoid evaporation. A $1 \mathrm{ml}$ aliquot was withdrawn at an interval of $0.5,1,2,3,4,5,6,7$ and $8 \mathrm{~h}$, and replaced with the same volume to maintain a sink condition. Samples were analyzed using spectrometric analysis (Kanabar et al., 2015; Sleigh et al., 2014).

Selection of population for trial. The human ethics committee of the Department of Anesthesiology, Peking Union Medical College Hospital, China, had approved the protocol (ANE/0504/Peking) for the present trial. The structure, nature, and intention of the study were made clear to volunteers and/or parents (in case of children). The trial conducted here is controlled, randomized and contains 15 subjects (Table 2) in each group $(n=15)$. No subject is excluded from the study due to the reason of any suspected allergy to local anesthetics or especially ropivacaine.

The subjects were allocated randomly to each treatment group. A patch containing $25 \mathrm{mg}$ of ropivacaine was either directly applied to the skin (Group I) or with aid of the RED stack device. Both selected patches were comparable in all physical and chemical parameters, such as weight, the drug content, etc. The basic design and pattern of the study with the use of rating scales was explained during visits and examples were given to verify whether the subject is able to use a scale or not.

Application of RED stack ${ }^{\mathrm{TM}}$ system and patches to volunteers. The device was attached to the skin surface and patch as per the instructions given in the manual. The installation and operation of the system was done under the guidance and supervision of a health care professional. The application site was waxed in order to ensure proper adhesion of the device and patch to the skin. The movement of the application site was abandoned in order to loosen contact of the device (Fig. 1A) with the skin surface.

All of the patients were administered with their respective test or control samples at the same time, using the same experimental conditions. The effects were measured in terms of endpoints.

Table 2. Population distribution of children based on age, sex and body weight.

\begin{tabular}{lll}
\hline Parameter & Group I & Group II \\
\hline Boys/Girls (mean \pm S.D.) & $7 / 8$ & $6 / 9$ \\
\hline Weight $(\mathrm{kg})$ (mean \pm S.D.) & $25.4 \pm 1.06$ & $24.12 \pm 1.65$ \\
\hline Age (years) (mean \pm S.D.) & $5.3 \pm 1.73$ & $7.1 \pm 1.63$ \\
\hline Pre-medication & None & None \\
\hline
\end{tabular}


$\mathbf{A}$

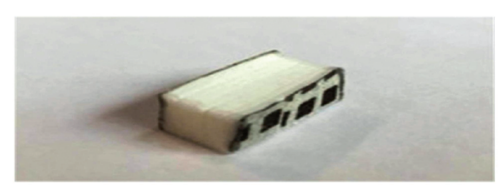

B
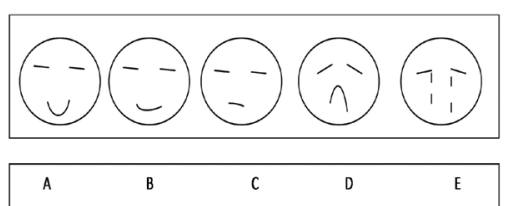

C

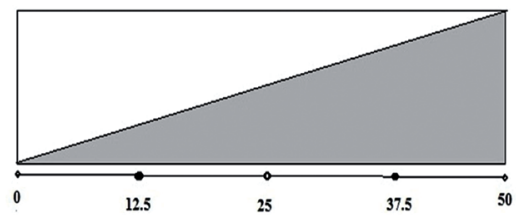

Figure 1. (A) Disposable RED system; (B) Visual scale showing intensity of pain as "no pain" for face A while "Unbearable pain" for face $E$. The pain intensity increases from $A$ to $E$; (C) Visual scale showing intensity of pain as "no pain" for point 0 while "Unbearable pain" for point 50 . The pain intensity increases from point " 0 " to point " 50 "

Efficacy endpoints. The intensity of pain sensed by subjects is based on endpoints when performed by a medical professional. The endpoints were measured at a time interval of $0,10,20,30,40,50$ minutes and 1 hour. Certain parameters, such as a warm sensation, cool sensation and hot pain were considered for measurement in the duration of anesthesia. The depth of anesthesia was also determined by measurement of a pain sensation using 27 gauge needles (Feng Liu et al., 2017).

The thermal threshold was measured in the central portion of the treated area. The stimuli from a lower to a higher order were measured using a meander electrode which consists of electrodes of alternating polarity where the gap is filled with an insulating material. The temperature of the thermode was either increased or decreased at a rate of $1.0^{\circ} \mathrm{C} /$ second, depending on the direction of the current flow through the device. This switching of temperature was done by the patients themselves.

The depth of anesthesia was assessed by inserting a 27-gauge short needle, perpendicular to the skin, in one direction with a controlled slow and smooth movement. The first sensation of pain was recorded on a visual scale 2 and scale 3. After the first sensation of pain, the needle was removed and the depth of insertion was marked. The surface of the skin was examined visually for any observable signs, such as redness, swelling, etc.

Measurement of endpoints on the scale. Visual scales were allocated in order to rate the intensity of pain experienced by subjects. These scales proved to be useful for subjective evaluation of pain in children. Scale 1 is framed at a hospital scale for very young children who might not be able to explain the level of pain felt by them. The left extreme part of Scale 1 (Fig. 1B), i.e.

Table 3. Physico-chemical evaluation of formulated injection batches.

\begin{tabular}{lllll}
\hline Batch & R1 & R2 & R3 & R4 \\
\hline pH & 4.9 & 5.1 & 5.2 & 4.8 \\
\hline Clarity & ++ & +++ & +++ & +++ \\
\hdashline Assay (\%) & 87.45 & 89.98 & 94.34 & 98.06 \\
\hline
\end{tabular}

face A, shows "no pain" at all while the rightmost part, i.e. face E, shows "unbearable pain".

Another visual scale, i.e. scale 2 (Fig. 1C), ranging as $50 \times 10 \mathrm{~cm}$ is used for evaluation of the intensity of pain based on numerical values. The scale shows numbers from 0 to 50 as a measure of pain. Here, 0 indicated "no pain" while 50 at the right extreme of scale is a marker of "unbearable pain". The scale is used for subjects who are capable of expressing the level of pain sensed by them. The patient tells the evaluator the number on a scale depending on the level of pain.

\section{RESULTS AND DISCUSSION}

Table 3 shows evaluation parameters for injection, while Table 4 contains evaluation for patches of ropivacaine. The solvent casting evaporation system was found to be successful in preparing patches of ropivacaine enclosing $25 \mathrm{mg}$ of the drug. Thus prepared patches were found to be comparable to the parameters set for the ideal transdermal system. Each prepared patch has exhibited good surface characteristic with a smooth appearance and semitransparent nature. The thicknesses of the patches were in the range of $0.02 \pm 0.006$ to $0.04 \pm 0.007 \mathrm{~mm}$, with $95.79 \pm 1.85$ to $97.45 \pm 0.07 \%$ drug content. The average weight and tensile strength was

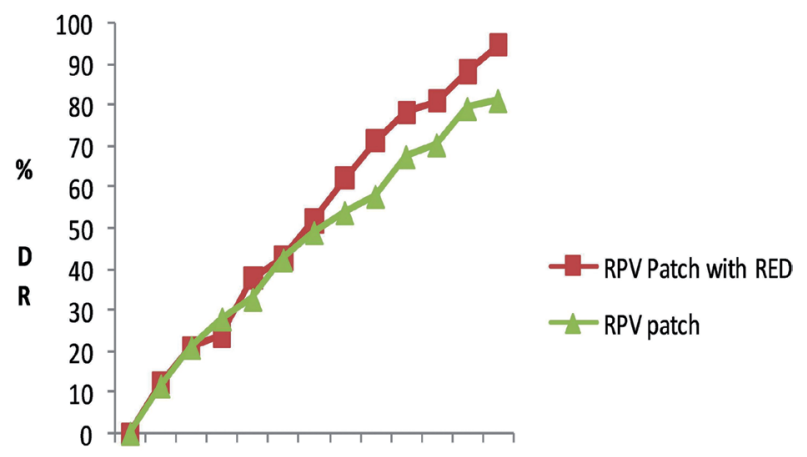

024681012141618202224

Time (HR)

Figure 2. In vitro drug release of the formulation with and without RED system attached.

The drug release from the patch with the RED system is faster when compared to the one without the RED system.

Table 4. Physico-chemical characterization of patches

\begin{tabular}{lllllllll}
\hline Batch & $\begin{array}{l}\text { Appe- } \\
\text { arance }\end{array}$ & $\begin{array}{l}\text { Thickness } \\
(\mathrm{mm})\end{array}$ & $\begin{array}{l}\text { Wt variation } \\
(\mathrm{g})\end{array}$ & $\begin{array}{l}\text { Folding } \\
\text { endurance }\end{array}$ & $\begin{array}{l}\text { Drug content } \\
\text { uniformity } \\
(\%)\end{array}$ & $\begin{array}{l}\text { Tensile } \\
\text { strength } \\
\left(\mathrm{kg} / \mathrm{cm}^{2}\right)\end{array}$ & $\begin{array}{l}\text { Moisture } \\
\text { absorption } \\
(\%)\end{array}$ & $\begin{array}{l}\text { Moisture } \\
\text { loss } \\
(\%)\end{array}$ \\
\hline $\begin{array}{l}\text { Group } \\
\text { I }\end{array}$ & $\mathrm{T}$ & $0.030 \pm 0.0041$ & $0.167 \pm 0.0017$ & $156.2963 \pm 2.2552$ & $95.89 \pm 0.91$ & $0.4258 \pm 0.0087$ & $1.4391 \pm 0.2521$ & $1.2154 \pm 0.2398$ \\
\hdashline $\begin{array}{lllllll}\text { Group } \\
\text { II }\end{array}$ & $\mathrm{S}$ & $0.045 \pm 0.0021$ & $0.159 \pm 0.0028$ & $163.3428 \pm 3.1243$ & $97.37 \pm 0.9185$ & $0.4856 \pm 0.0042$ & $1.8556 \pm 0.2143$ & $2.3932 \pm 0.2732$ \\
\hline
\end{tabular}

T, Transparent; S, smooth; R, Rough 
Table 5. Effect of the RED system on the skin of the subjects

\begin{tabular}{lll}
\hline & Group I & Group II \\
\hline Swelling & Negative & Negative \\
\hdashline Itching & Mild & Mild to Not at all \\
\hline Edema & Negative & Negative \\
\hline
\end{tabular}

Table 6. Mean pain intensity score assessment in patients using visual analytical scale (VAS) after treatment with each test formulation.

\begin{tabular}{llc}
\hline \multirow{2}{*}{ Time $(\min )$} & \multicolumn{2}{l}{ Mean Pain Intensity Score (VAS 0-10) } \\
\cline { 2 - 3 } & Group I & Group II \\
\hline 0 & 7.9 & 7.2 \\
\hline 5 & 7.4 & 6.4 \\
\hline 10 & 6.2 & 5.4 \\
\hline 15 & 4.5 & 3.2 \\
\hline 20 & 4.1 & 2.4 \\
\hline 25 & 4 & 0 \\
\hline 30 & 3.6 & 0 \\
\hline
\end{tabular}

found to be $0.15 \pm 0.2$ to $0.17 \pm 0.18 \mathrm{gm}$, and $0.42 \pm 0.006$ to $0.58 \pm 0.002 \mathrm{~kg} / \mathrm{cm}^{2}$, respectively. The folding endurance of the patches was in the range of $155.21 \pm 0.2$ to $167 \pm 0.29$, and the moisture content was $1.43 \pm 0.23 \%$ to $2.97 \pm 0.23 \%$. The formulation shows an in vitro release in the phosphate buffer ( $\mathrm{pH} 7.4$ ) as $55.56 \%$ to $89.23 \%$ \pm 0.24 in $24 \mathrm{~h}$ with the reverse electrodialysis system attached, and $43.76 \%$ to $81.23 \% \pm 0.14$ in $24 \mathrm{~h}$ without the reverse electrodialysis system (Fig. 2).
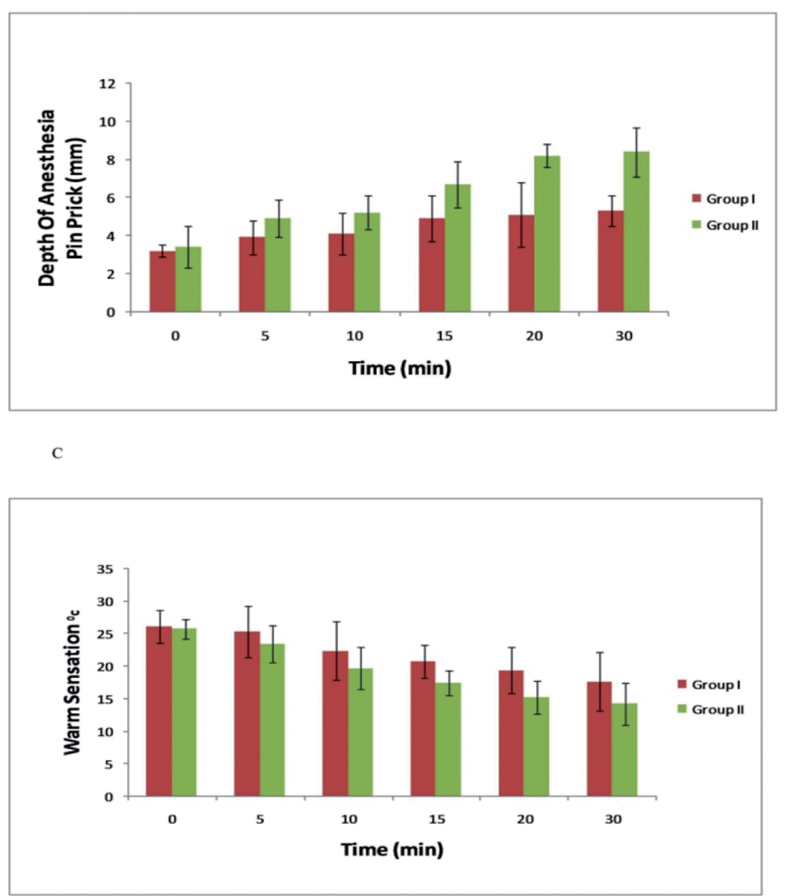

Initially, 33 subjects were selected for the study. However, one child was removed from group II before attaching assembly of patch and RED due to their nonco-operative and anxious nature. Another two subjects were removed (one from each group) due to inability to respond to the method of experiment. In total, 30 subjects were enrolled for this study, including both boys and girls in different ratios, with an average age of 4-7 years and body weight of 19-26 kg (Table 2). No subject entered in the trial was kept on any type of medication.

All of the subjects who underwent treatment (both in Group I and Group II) have shown no signs of swelling, itching or edema, although some of them reported mild irritation or a tingling sensation which was considered normal Table 5.

The time lapse for pain intensity for both groups is shown in Table 6. The pain intensity after treatment for Group I (with ropivacaine patch only) was found to be higher than that for group II (ropivacaine patch with the RED system). The pain was subdued comparatively faster in group II than in Group I.

Initially, for both groups, the pain intensity at baseline or at 0 was numerically the same or comparable, but with increasing time the subjects in Group II reported the lesser sensation of pain, while those in group I took more time. More precisely, after 20 minutes of administration, the subjects in group II reported complete numbness i.e. "no pain"; at the same time, the subjects in group I still reported a sensation of some pain, even after 20 and 30 minutes. Thus, this numerical difference in intensity score shows the superiority of the transdermal patch when administered with aid of reverse electrodialysis.

In case of the cool sensation, as can be seen in Fig. 3, at $0 \mathrm{~h}$ there was no significant difference between the two test groups, but as the treatment time had passed,
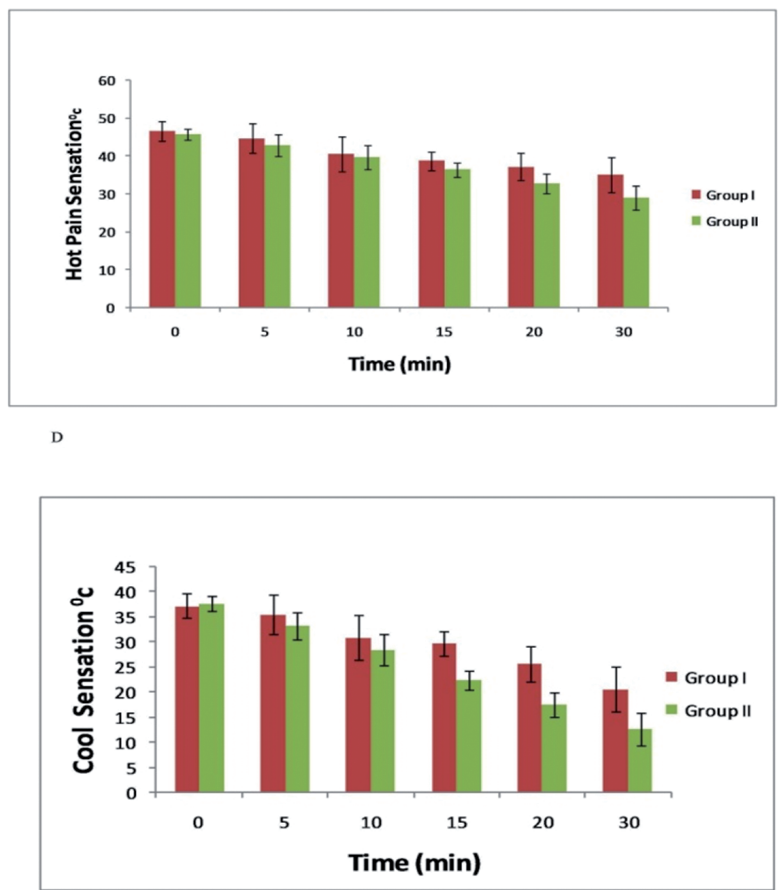

Figure 3. Graphical representation of endpoint measurements;

(A) Depth of Anesthesia: depth of anesthesia is much deeper for group II; (B) Hot pain sensation: (group I) shows observation of the highest pain intensity throughout the time course; (C) Warm sensation: (group I) shows observation of the highest pain intensity throughout the time course; (D) Cool Sensation: This activity is increased in the case of group II when compared to group I. 
the ability of formulation with the RED system to combat the cool sensation had increased, i.e. the anesthetic effect had increased. This enhanced effect had helped to reduce the cool sensation. This activity seems to be increased in case of group II when compared to group I.

The effect on depth of anesthesia can be seen in Fig. 3. It measures the distance in $\mathrm{mm}$ up to which the needle can be inserted. From Fig. 3 one can clearly state that the depth of needle pricks is highest for group II which means the patients are more anesthetized in this group and indicates that the depth of anesthesia is much deeper for group II.

The VAS scale i.e. visual analytical scale was used for the measurement of the intensity of pain. The study undertaken here is controlled and randomized with the concentration of local anesthetic kept constant. The duration of the study for both groups was also kept constant. The present investigation also focuses on in vitro release of drug when the patch is fixed to reverse electrodialysis system. The investigation proves the efficacy of the RED system over the normal patch in the increasing rate of release of drug from patch (http://www. redstack.nl/en/technology/reverse-electrodialysis-red).

The physical responses obtained from the patients in the present trial showed variation and a distinct pattern. The patients in the treatment group without the RED system (group I) experienced the highest pain intensity in the time course, as there was no effect of an anesthetic. The initial mean pain intensity observed for treatment group 2 shows approximately the same level of pain intensity as group 1 , but rapidly decreases as the time for treatment passes. When compared to the treatment group 2, group 1 shows lesser lowering of pain intensity. Cool sensations are results of response by small myelinated fibers, while warmth and pain sensations are due to small unmyelinated fibers. The study undertaken here supports the fact that myelinated fibers are more sensitive to the local anesthetic blockade (http://www. redstack.nl/en/technology/reverse-electrodialysis-red, Feng Liu et al., 2017)

\section{CONCLUSION}

The study presented here shows a successful formulation of ropivacaine patches, using a solvent casting evaporation method. It also proves that ropivacaine patches, when applied to children of various age group of both sexes with aid of reverse electrodialysis system, show superiority in lowering the pain threshold and cool sensation when compared with patch application only.

\section{REFERENCES}

Amnuaikit C, Ikeuchi I, Ogawara K, Higaki K, Kimura T (2005) Skin permeation of propranolol from polymeric film containing terpene enhancers for transdermal use. Int J Pharm 289: 167-178. https:// doi.org/10.1016/j.ijpharm.2004.11.007

Araujo DR, Cereda CM, Brunetto GB, Vomero VU, Pierucci A, Neto HS, de Oliveira AL, Fraceto LF, Braga Ade F, de Paula E. (2008) Pharmacological and local toxicity studies of a liposomal formulation for novel local anesthetic ropivacaine. J Pharm Pharmacol 60: 1449-1457. https://doi.org/10.1211/jpp/60.11.0005

Cederthoim I (1997) Preliminary risk-benefit analysis of Ropivacaine in labor and following surgery. Drug Safety 16: 391-402. https://doi. org/10.2165/00002018-199716060-00005

Chen H, Wang Y, Zhai Y, Zhai G, Wang Z, Liu J (2015) Development of ropivacaine loaded nanostructured lipid carrier formulation for transdermal delivery. Colloids Surfaces A: Physicochem Eng Aspects 465: 130-136. https://doi.org/10.1016/j.colsurfa.2014.10.046

Feng Liu, Lu Liu (2017) Iontophoretic delivery of lignocaine in healthy volunteers: effect of concentration of epinephrine on local anesthesia. Biomedical Res 28: 185-191

Green SM (2000) Modern anesthesiologists receive limited training with Ropivacaine - Implications for emergency medicine. Acad Emerg Med 7: 839-841. https://doi.org/10.1111/acem.13619

http://www.redstack.nl/en/technology/reverse-electrodialysis-red

https://en.wikipedia.org/wiki/Transdermal_patch

https://prosoluspharma.com/about-transdermals

Jett WE (2018) Three Generations: The Past, Present, and Future of Transdermal Drug Delivery Systems.

Kanabar Vishvesh B, Patel Vipul P, Doshi Sumit M (2015) Formulation and evaluation of transdermal patch of Cefdinir with various polymers. The Pharma Innovation J 4: 74-77

Mazzenga GC, Berner B (1991) Transdermal delivery of zwitterionic drugs I: the solubility of zwitterion salts. J Control Release 16: 77-88. https://doi.org/10.1016/0168-3659(91)90032-9

Morales Moraes C, Prado de Matos A, de Lima R, Rosa AH, de Paula E, Fraceto LF (2007) Initial Development and characterization of PLGA nanospheres containing Ropivacaine. J Biolphys 33: 455-461. https://doi.org/10.1007/s10867-008-9094-z

Pattle RE (1954) Production of electric power by mixing fresh and salt water in the hydroelectric pile. Nature 174: 660-672. https://doi. org/10.1038/174660a0

Post JW, Hamelers HVM, Buisman CJN (2008) Energy recovery from controlled mixing salt and fresh water with a reverse electrodialysis system. Environ Sci Technol 42: 5785-5798

Rawat S, Vengurlekar S, Rakesh B, Jain S (2008) Transdermal Delivery by Iontophoresis. Indian J Pharm Sci 70: 5-10. https://doi.org/ 10.4103/0250-474X.40324

Reeves JG, Glass PS, Lubarsky DA, McEvoy MD, Ruiz RM (2010) Intravenous anesthetics. In Miller's Anesthesia, Miller RD, eds, 7th edn, pp 719-71 USA: Churchill Livingstone

Sachanand R, Bajpai M (2013) Transdermal drug delivery system: a review. Int J Res Dev Pharm Life Sci 3: 748-765

Sleigh J, Harvey M, Voss L (2014) Denny Ropivacaine-more mechanisms of action than just NMDA blockade. Trends Anaesth Crit Care 4: 76-81. https://doi.org/10.1016/j.tacc.2014.03.002 2210-844

Tyle P (1986) Iontophoretic devices for drug delivery. pp 318-326. American Cyanamid Company. New Jersy

Veerman J, Saakes M, Metz SJ, Harmsen GJ (2009) Reverse electrodialysis: performance of a stack with 50 cells on the mixing of sea and river water. J Membr Sci 327: 136-149. https://doi.org/10.1016/j. memsci.2008.11.015

William T, Zempsky MD, Anand KJS (1998) Lidocaine iontophoresis for topical anesthesia before intravenous line placement in children. J Pediatrics 12: 1061-1063 